\title{
An 80 x 25 Pixel CMOS Single-Photon Range Image Sensor with a Flexible On-Chip Time Gating Topology for Solid State 3D Scanning
}

\author{
Henna Ruokamo, Lauri Hallman, Harri Rapakko and Juha Kostamovaara \\ Faculty of Information Technology and Electrical Engineering, \\ Circuits and Systems Research Unit, \\ University of Oulu, Finland \\ henna.ruokamo@oulu.fi
}

\begin{abstract}
A solid state 3D scanner based on a pulsed laser diode source and narrow time gating of a 2D CMOS single photon avalanche diode (SPAD) detector array is presented. The imager uses an on-chip delay-locked loop to program the time gating of 40 sub-arrays individually. The prototype detector has $80 \times 25$ pixels with a fill factor of $32 \%$ in the sensor area. The chip has been fabricated in a $0.35 \mu \mathrm{m}$ highvoltage process and occupies a $5.69 \times 5.02 \mathrm{~mm}^{2}$ area. A 3D range image rate of $\sim 5$ frames/second with centimeter level precision is demonstrated to passive targets within a range of $\sim 1$ meter and FOV of $36 \times 57$ degrees.
\end{abstract}

Keywords-3D scanner; single-photon avalanche diode (SPAD); time gating; SPAD Array

\section{INTRODUCTION}

Three dimensional imaging of the environment is important for autonomous driving systems, robotic vision and surveillance applications, for example. A low-price and small 3D-scanner would find use also in human-machine interfaces and gaming applications.

A promising technique to realize a small and robust 3Dscanner is to use the optical time-of-flight (TOF) technique with the electronic focal plane scanning approach. In this technique, a laser diode source illuminates the field of view of a single photon avalanche diode array. When the detector array is located at the focal plane of a positive lens, a 3D range image of the field-of-view of the system can be produced without any rotating mirrors or other moving parts. The distance in TOF systems is measured either directly from the time intervals taken by the photons to travel from the laser transmitter to the target and back to the receiver or indirectly by utilizing a phase comparison technique with a continuous wave $(\mathrm{CW})$ modulated source.

With the phase comparison technique, a measurement range of $0.8-4.2 \mathrm{~m}$ with fairly good accuracy of $<1 \%$ has been achieved [1]. However, the measurement range achieved with this technique is typically short. Another typical realization utilizes a pulsed laser source, which allows for high single shot precision and in many cases also wider measurement range, even km's with high enough transmitter energy and single photon detection mode [2].
The pulsed TOF range finding method usually uses the direct distance measurement technique with a time-to-digital or time-to-amplitude converter and can achieve a millimeter level accuracy even to a range of more than a few tens of meters [3]. The drawback of this technique is the sensitivity to background light if the sensor is open for photons during a time window covering the whole measurement range (e.g. $100 \mathrm{~ns}$ for a measurement range of $15 \mathrm{~m}$ ). With strong ambient light conditions, one solution is to use a fast electric shutter to control the sensor's sensitivity to photons by limiting the time interval during which the detectors are enabled for the photon detection [2 - 5]. In this work, the width of the time gating with an electric shutter is set to the minimum and thus the imager is very tolerant to background light.

The fill factor of the sensor array should be high in the focal plane approach to maximize the photon detection efficiency. The larger the SPAD array, the more difficult it is to get a high fill factor due to the large number of routing signals or due to in-pixel electronics. Typically, large arrays with more than 1000 pixels tend to have a small, less than $10 \%$ fill factor [4].

In this work, we propose a CMOS SPAD imager topology utilizing narrow and flexible time gating of a $80 \times 25$ pixel SPAD array, and show also the first 3D range image measurement results achieved with this device. Although time gating has been successfully implemented in single photon detector arrays before [5], this kind of a programmable and separable time gating of pixel sub-arrays together with the use of a laser diode transmitter with high energy $(\sim 1 \mathrm{~nJ})$ sub-ns pulses is suggested here for the first time for $3 \mathrm{D}$ range imaging, to the best knowledge of the authors. The flexibility of the time gating is a remarkable advantage since it enables the sub-arrays of the sensor area to be individually set to measure (or scan) different distance windows. The use of sub-ns laser pulses ( 100 ps FWHM) allows for a good single shot resolution at the level of $1-$ $2 \mathrm{~cm}$.

In the following, the operating principle of the proposed $3 \mathrm{D}$ imager is described first. Then the pixel design and architecture of the chip are described. At the end of the 
paper, the first $3 \mathrm{D}$ range image measurement results are shown.

\section{3D RANGE IMAGER OPERATING PRINCIPLE}

The 3D range imager proposed here is based on the pulsed TOF technique and flexible time gating of the SPADs in the detector array. The detector chip collects binary type 2D cross-sectional images from predetermined distances which are defined by the time gating electronics, see Fig. 1. The chosen measurement range is scanned, and the $3 \mathrm{D}$ image is accumulated from the $2 \mathrm{D}$ cross-sectional images of the surface of the target resulting from the single shot measurements.

The principle of setting the time gates of sub-arrays at different time (range) locations is shown in Fig. 2. In the prototype circuit, the pixel array is divided into 40 subarrays, each of which has $10 \times 5$ SPADs. The position of the time gates can be programmed individually for each subarray within the total time window of $24 \mathrm{~ns}$, which corresponds to a range of $\sim 3.6 \mathrm{~m}$. The width of the time gates can be set larger when searching for a target and decrease to the minimum when the target is found and the highest resolution is needed. The partitioning of the large sensor array into sub-arrays increases the total 3D frame rate of the system since partial scans around the surface of the target or even multiple targets at different distances can now be implemented without the need to scan over the whole image space and depth.

With a pulsed laser source that has high energy $(>1 \mathrm{~nJ})$ and narrow full width at half maximum (FWHM $\sim 100 \mathrm{ps}$ ), a single-shot depth resolution of $<2 \mathrm{~cm}$ is achievable [6]. The detector time measurement precision depends on the SPAD timing jitter ( $50-100$ ps typically) and on the time gating precision. The shifting of the time gate position with respect to each other can be done on-chip in a grid of $\sim 100 \mathrm{ps}$ (LSB) in the prototype. The length and position of the time gates are selected from altogether 240 outputs of two on-chip delay-locked loops. The selection of the time gate for each sub-array is controlled by the FPGA.

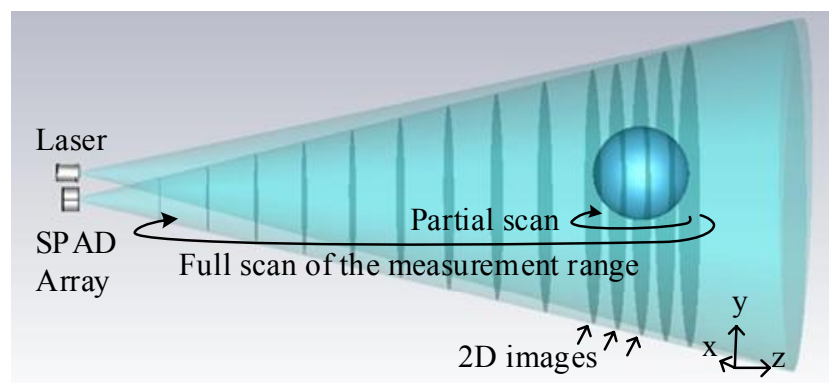

Fig. 1. 3D imaging with cross-sectional 2D images.

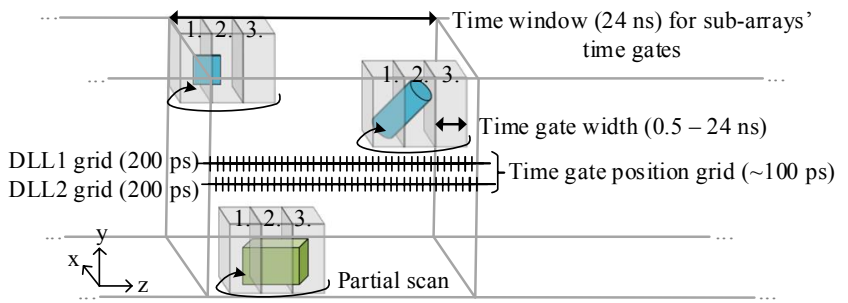

Fig. 2. The 2D pixel array is divided in to $2 \mathrm{D}$ sub-arrays whose time gating can be programmed individually.

The laser driver is controlled from the detector chip. In this way the measurement range can be set to begin right at the lens of the detector.

\section{PIXEL ELECTRONICS}

In order to get a high fill factor for the sensor part of the chip, the electronics inside the sensor array have been minimized. Biasing switches, 1-bit data storage, and buffering of signals were implemented inside each pixel. A simplified schematic of the pixel is presented in Fig. 3.

At the beginning of the operation, the SPAD is quenched by biasing the anode node of the diode to the voltage supply of $3.3 \mathrm{~V}$. The cathode of the diode is connected to a highvoltage supply of $\sim 22 \mathrm{~V}$. When the quenching switch is turned off, the anode node can be shunted to the ground. This forms the leading edge of the time gate and exposure of the sensor for photons.

The timing diagram of the pixel electronics is presented in Fig. 4. The load signal for enabling the SPAD is produced locally at each pixel from the rising edges of load start and load stop signals coming from outside the sensor array. By transferring signal information in rising edges makes it easier to produce fast and narrow loading pulses to the other side of the large array. In this case, for example, the longest trace lengths are over $2.5 \mathrm{~mm}$. The trailing edge of the time gate is produced by the leading edge of sample signal which samples the state of the SPAD into the 1-bit memory cell. After the states of all the SPADs in the array have been sampled, the flip-flops are connected in series and the measurement data is read out from the pixels in series mode.

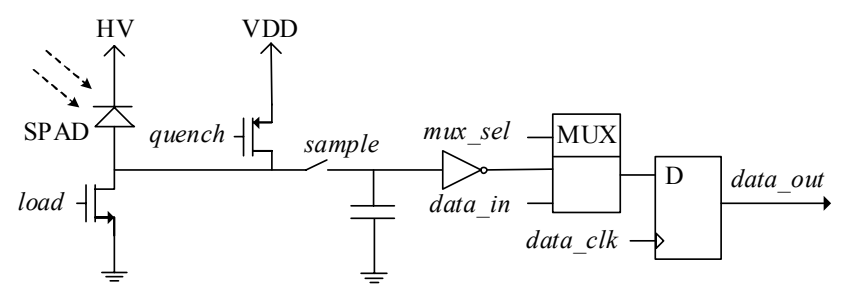

Fig. 3. Schematic of the pixel electronics. 


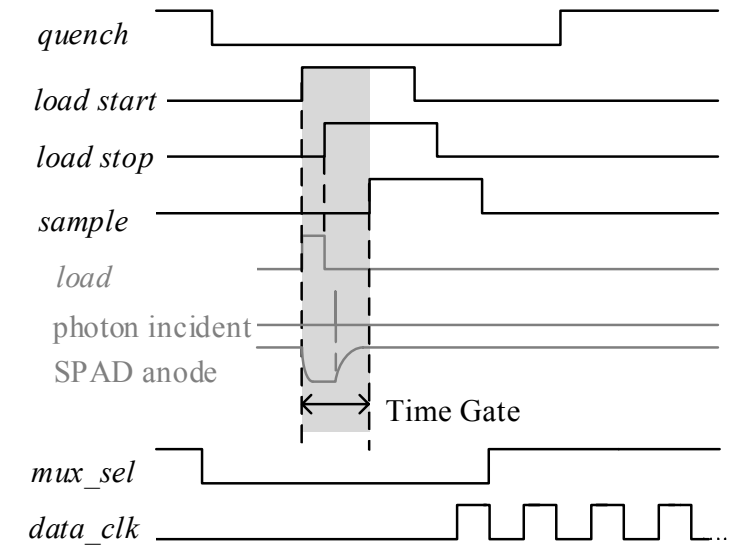

Fig. 4. Timing diagram of pixel electronics.

The size of one pixel is $50 \mu \mathrm{m} \times 100 \mu \mathrm{m}$ and the active area of one SPAD in a pixel is $36 \mu \mathrm{m} \times 44 \mu \mathrm{m}$. This results in an average fill factor of $\sim 32 \%$ for the whole sensor area. A relatively large SPAD pixel area was chosen to get a high fill factor. The resulting higher noise (DCR) due to the larger SPAD area is not an issue due to the narrow time gates used. The multiplication region of the avalanche diode is produced by a deep nwell $/ \mathrm{p}+$ junction and the active area is surrounded by pwell guard rings as in [7]. The SPADs are placed in rows of 80 pixels which all share the same deep nwell. Also, every other of the 25 rows is flipped in the ydirection and placed in the same deep nwell to increase the fill factor.

\section{DETECTOR ARCHITECTURE}

The detector prototype consists of a detector array of $80 \times 25$ pixels and programmable on-chip time gating, control and data processing with an FPGA, see Fig. 5.

The time gating signals are produced on-chip from two delay-locked loops (DLLs) each of which has 120 outputs. The length of both DLLs is $24 \mathrm{~ns}$ with an $83.3 \mathrm{MHz}$ clock signal from the FPGA.

The independent time gating signals for each of the 40 sub-arrays can be selected from the DLL outputs with multiplexers. Hence, time gates at each sub-array can vary with the maximum of $\sim 24 \mathrm{~ns}$ in relation to each other. In the prototype, each of the time gates are produced from three independently selectable signals. The selection of the signals is made with 240-to-1 multiplexers (MUX) from the DLLs' 240 outputs. Each of these $3 * 40$ MUXs is controlled by the external FPGA with 8 bits.

In order to be able to place the time window of $24 \mathrm{~ns}$ (see Fig. 2) to the desired time position, a control block was implemented. This control block enables the DLLs' output buffers for a specific time window. The control block has a 6-bit counter which counts total DLL cycles after the laser command has been sent. The control block is controlled by the FPGA with 6 bits and enables a time window of $24 \mathrm{~ns}$ for the time gating even with a $\sim 1.5 \mu$ s delay from the laser pulse.

To be able to measure distances from zero distance, a selectable delay for the laser shoot command has been implemented. The selection is done from one of the 120 DLL outputs with the delay grid of $200 \mathrm{ps}$. The signal for laser driver circuitry is buffered in the same way as the time gate signals to reduce the effect of temperature and supply voltage changes.

The state of the 2000 SPADs at the trailing edge of the time gate is sampled into in-pixel memory cells. The result is then read out by connecting 80 of these 1-bit registers serially and buffering all the $80 \times 25$ bits to an external FPGA for further processing. With a $100 \mathrm{MHz}$ readout clock, up to $1 \mathrm{MHz}$ frame rate for single measurement shots can be achieved. The transfer of all of the data to the external FPGA makes the signal processing flexible especially for a prototype version, but at the cost of larger power consumption due to the need of multiple large high speed buffers.

The prototype of the detector chip was fabricated in a cost-effective $0.35 \mu \mathrm{m}$ high-voltage process and the dimensions of the chip are $5.02 \mathrm{~mm} \times 5.69 \mathrm{~mm}$. The picture of the chip and the layout of one sub-array of pixels are presented in Fig. 6. The layout of the chip is designed so that the array size can be multiplied by two or even four by pipelining the registers and rearranging the power supply pins.

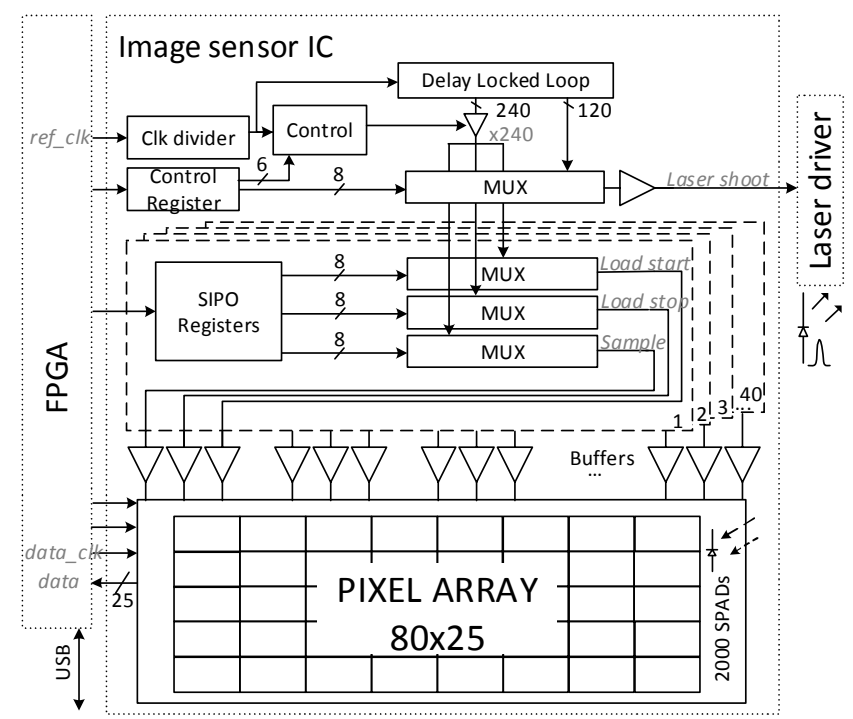

Fig. 5. Block-level representation of the imaging system. Note separate timing controls for 40 SPAD sub-arrays.

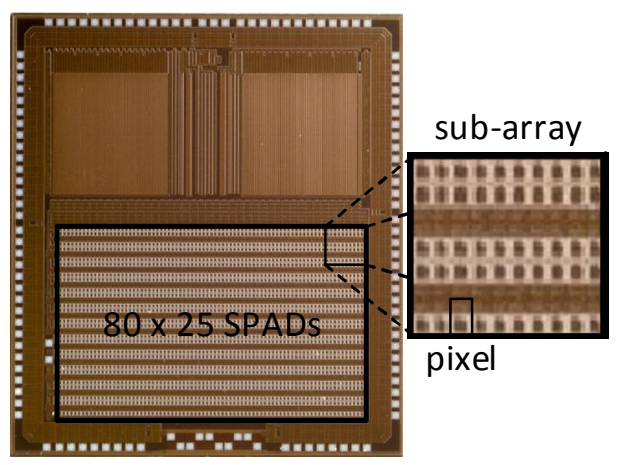

Fig. 6. Picture of the chip. 


\section{MEASUREMENT Results}

A 3D range image of two spheres is shown in Fig. 7. The diameters of the targets with non-cooperative surfaces are $30 \mathrm{~cm}$ and $11 \mathrm{~cm}$ and they were placed at the distances of $80 \mathrm{~cm}$ and $60 \mathrm{~cm}$ from the transceiver. The positions of the time gates at the beginning of the scan are shown in Fig. 7 a) and a partial scan range of $30 \mathrm{~cm}$ with $\sim 1.5 \mathrm{~cm}$ $(\sim 100 \mathrm{ps})$ LSB $\mathrm{z}$-resolution was selected. The time gate widths were $800 \mathrm{ps}$. The shown result was achieved by using 1000 laser pulses for each of the time gate positions (i.e. 20000 laser pulses for the $30 \mathrm{~cm}$ partial scan). The laser pulsing rate was $100 \mathrm{kHz}$ resulting in a 3D frame rate of $5 \mathrm{~Hz}$. The laser pulse had a wavelength of $860 \mathrm{~nm}$, an energy of $\sim 1 \mathrm{~nJ}$ and FWHM of $110 \mathrm{ps}$. An optical bandpass filter was placed in front of the detector lens to suppress the ambient light. The measured ambient lighting at the object FOV was 200-600 lux. The current consumption of the receiver from a $3.3 \mathrm{~V}$ power supply is $20 \mathrm{~mA}$ of which $5 \mathrm{~mA}$ is consumed by the delay-locked loops and the time gating circuitry.

\section{CONCLUSIONS}

A time gating topology for solid state $3 \mathrm{D}$ scanning with a CMOS SPAD imager is presented. The topology is based on two on-chip delay-locked loops which have altogether 240 outputs from which the time gating signals can be selected. The 2D SPAD array has been divided into 40 sub-arrays whose time gating can be programmed individually from the delay line outputs with 8 bits. The measurement results show functionality of the topology and the detector's insensitivity to normal office lighting with 800 ps time gate widths. The fabricated imager has $80 \times 25$ pixels in a sensor area of $4 \mathrm{~mm} \times 2.5 \mathrm{~mm}$ with a $32 \%$ fill factor which is remarkable for a large $(>1000)$ SPAD array without the use of micro lenses. A 3D range image rate of $\sim 5 \mathrm{fr} / \mathrm{sec}$ with $\sim \mathrm{cm}$ precision is demonstrated to passive targets within a range of $\sim 1 \mathrm{~m}$ and $36 \times 57$ degrees FOV. The result compares quite favorably with the state of the art [8] taking into account the large FOV and much lower illumination power (average $0.1 \mathrm{~mW}$ ) and receiver aperture $(\sim 3.5 \mathrm{~mm})$.

\section{ACKNOWLEDGMENT}

The authors acknowledge financial support from the Academy of Finland (Centre of Excellence in Laser
Scanning Research) and the Infotech Oulu Graduate School.

\section{REFERENCES}

[1] C. S. Bamji et al., "A $0.13 \mu \mathrm{m}$ CMOS System-on-Chip for a $512 \times 424$ Time-of-Flight Image Sensor With MultiFrequency Photo-Demodulation up to $130 \mathrm{MHz}$ and $2 \mathrm{GS} / \mathrm{s}$ ADC," in IEEE Journal of Solid-State Circuits, vol. 50, no. 1, pp. 303-319, Jan. 2015.

[2] M. Perenzoni, D. Perenzoni and D. Stoppa, "A $64 \times 64-$ Pixels Digital Silicon Photomultiplier Direct TOF Sensor With 100-MPhotons/s/pixel Background Rejection and Imaging/Altimeter Mode With 0.14\% Precision Up To $6 \mathrm{~km}$ for Spacecraft Navigation and Landing," in IEEE Journal of Solid-State Circuits, vol. 52, no. 1, pp. 151-160, Jan. 2017.

[3] S. Jahromi, J-P. Jansson, and J. Kostamovaara, "Solidstate $3 \mathrm{D}$ imaging using a $1 \mathrm{~nJ} / 100 \mathrm{ps}$ laser diode transmitter and a single photon receiver matrix," Opt. Express 24, 21619-21632 (2016)

[4] D. Bronzi, F. Villa, S. Tisa, A. Tosi and F. Zappa, "SPAD Figures of Merit for Photon-Counting, PhotonTiming, and Imaging Applications: A Review," in IEEE Sensors Journal, vol. 16, no. 1, pp. 3-12, Jan.1, 2016.

[5] S. Burri, Y. Maruyama, X. Michalet, F. Regazzoni, C. Bruschini, and E. Charbon, "Architecture and applications of a high resolution gated SPAD image sensor," Opt. Express 22, 17573-17589 (2014).

[6] J. Kostamovaara, J. Huikari, L. Hallman, I. Nissinen, J. Nissinen, H. Rapakko, E. Avrutin, and B. Ryvkin, "On laser ranging based on high-speed/energy laser diode pulses and single-photon detection techniques," IEEE Photonics J. 7(2), $1-15$ (2015).

[7] M. Perenzoni, L. Pancheri, and D. Stoppa, "Compact SPAD-based pixel architectures for time-resolved image sensors," Sensors 16(5), 745 (2016).

[8] F. Villa et al., "CMOS Imager With 1024 SPADs and TDCs for Single-Photon Timing and 3-D Time-of-Flight," in IEEE Journal of Selected Topics in Quantum Electronics, vol. 20, no. 6, pp. 364-373, Nov.-Dec. 2014.
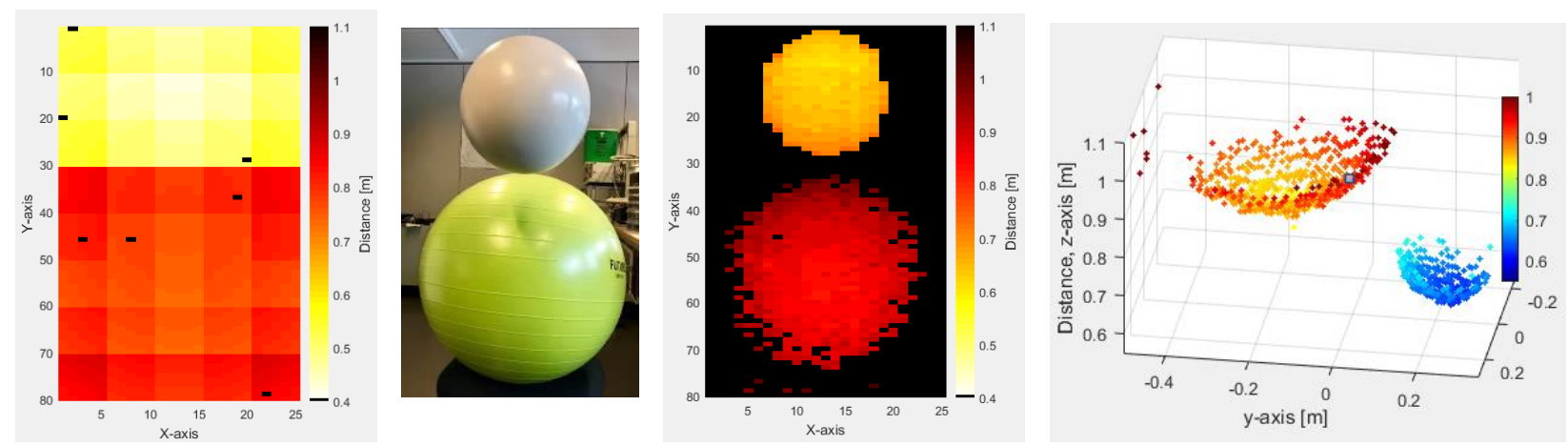

Fig. 7. a) Position of the time gates and eliminated noisy pixels at the start of the scan, b) picture of the targets, c) distance result of the pixels and d) 3D image of the targets. 